$\alpha_{V}$ Integrins 10535

Acute myeloid leukemia 11629

Acute myeloid leukemia 8-9 440

Adeno-associated virus 391

Adenocarcinoma of the esophagus 7366

Adjuvant therapy 1-2 19

Adults 12657

All-trans retinoic acid $\mathbf{1 1} 629$

Allogeneic stem cell transplantation 12691

Anal cancer $\mathbf{5} 251,5271$

Anastrozole 1-2 19

Angioimmunoblastic T-cell lymphoma 10546

Antiangiogenesis 10550

Anti-CD20 antibody rituximab 12691

Antineoplastic combined chemotherapy protocols $\mathbf{1 2} 665$

Apoptosis $\mathbf{1 2} 702$

Aromatase inhibitors 1-2 19, 6328

Arteriovenous malformations $\mathbf{6} 328$

Axillary lymph node dissection $\mathbf{6} 321$

Bevacizumab 7 391, 7 394, 8-9 464

Bilateral breast cancer $\mathbf{1 0} 542$

Biliary tract cancer $\mathbf{5} 271$

Bisphosphonates 10521

Bone Marrow Transplantation 5266

Bone metastases $\mathbf{1 0} 521$

Bortezomib 1-2 45

Brain metastases $\mathbf{3} 123$

Brain metastases: prognostic factors, age 6305

Breast 12653

Breast cancer 1-2 19, 3 99, 7 387, 10529 , 12685

Breast cancer, triple negative $\mathbf{1 1} 610$

Breast conserving surgery $\mathbf{3} 99$

Breast neoplasms 6328

Burden of illness 8-9 477

Cancer 5243

Cancer of the neovagina $\mathbf{1 - 2} 53$

Cardiac tamponade $\mathbf{1 0} 546$

Catheter migration 8-9 455
Central venous catheter 5262

Central venous catheter complications 11605

Cerebral infarct 3115

Cetuximab 8-9 464

Chemoradiotherapy 8-9 435

Chemotherapy 3 119, 7 391, 7394 , 8-9 469, 10 535, 12657

Chronic lymphocytic leukemia 4197

Chronic myeloid leukemia $\mathbf{1 1} 585$

Chylous leakage: diagnosis, treatment 6321

Cisplatin 12673

Colorectal cancer $\mathbf{5}$ 237, 5 271, 7394 , 8-9 464

Complete response $\mathbf{6} 315$

Computed tomography $\mathbf{1 - 2} 37$

Cost-effectiveness analysis $\mathbf{3} 85$

Costs of illness 385

Crossover studies 8-9 447

C-reactive protein $\mathbf{1 2} 665$

Cystitis 7391

Derivative chromosome $9 \mathbf{1 1} 585$

Dexamethasone 6309

Distress screening 11591

Docetaxel 1-2 11, 8-9 447

DSM-IV 6343

EBV 12691

EGFR 4174

EGFR inhibition $\mathbf{3} 123$

EGFR tyrosine kinase inhibitors 5237

Elderly patients 6335

Electric fields 7362

Endorectal MRI 5230

Endorectal ultrasound 5230

Enophthalmus 10529

EORTC QLQ-PR25 11599

Epithelial ovarian cancer $\mathbf{6} 315$

Erlotinib 3123

Erythrocyte transfusion 8-9 477

Esophageal cancer 11 620,12 673

Estrogen receptor modulators $\mathbf{6} 328$

Ewing's sarcoma, adults 4179

Exemestane 1-2 19
Exfoliative dermatitis $\mathbf{1 1} 616$

Exfoliative toxins $\mathbf{1 1} 616$

Expert rating scale $\mathbf{1 1} 591$

Extramedullary myeloma localization 1-2 45

Extraocular metastases $\mathbf{7 3 8 7}$

Extravasation 5262

Ewing's tumor 12657

FDG-PET 4166

FDG-PET-CT 8-9 474

FISH 391

Fludarabine, oral 4197

5-Fluorouracil 12673

Fluorescence in situ hybridization (FISH) 8-9 440

Fluorescence in situ hybridization 11585

Fluorodeoxyglucose, 18F 3107

FOXP3 5243

Fulminant hepatitis $\mathbf{1 1} 620$

Gastric cancer 5271

Gastrointestinal tumours 5271

Gefitinib 4 174, 5237

Gemcitabine 1-2 11

Geriatric assessment $\mathbf{6} 335$

Geriatrics 8-9 477

Giant cell arteritis of the breast $\mathbf{1 2} 685$

Glioblastoma: Extraneural spread, Bone, Lung, Soft tissue 4192

Glioma, malignant $\mathbf{6} 309$

Gynecological cancer 7 375, 11625

\section{HAART 7394}

HDAC inhibitors $\mathbf{1 1} 629$

Health-related quality of life $\mathbf{1 1} 599$

Hepatectomy 7375

Hepatocellular carcinoma $\mathbf{1 0} 550$

Hepatomegaly 6325

Herpes simplex virus $\mathbf{1 1} 620$

Histology 10521

History of medicine $\mathbf{1 2} 702$

HIV 7394

Hodgkin's lymphoma 1-2 49

Home parenteral nutrition $\mathbf{1 1} 605$

\title{
KARGER
}

(C) 2008 S. Karger GmbH, Freiburg 
Hydro-CT 5230

Hydroxymethylglutaryl-CoA reductase inhibitors 7391

Hyperbaric oxygenation 7391

Ibritumomab tiuxetan 1-2 49

Immunohistochemistry 4179, 10535

Immunomodulation 1-2 28

Immunosuppression $\mathbf{5} 243$

Immunotherapy 1-2 49

Interferon 1-2 28

Invasive ductal carcinoma $\mathbf{1 2} 685$

Inversion 16 8-9 440

Irinotecan $\mathbf{5} 237$

Köbner's phenomenon 6332

KRAS 4174

Leiomyosarcoma 1-2 11

Letrozole 1-2 19

Lichen ruber $\mathbf{6} 332$

Liver metastasis 7375

Liver resection $\mathbf{7 3 7 5}$

Long-term course 6343

Long-term survival $7380, \mathbf{8 - 9} 469$

Lung cancer $\mathbf{6} 305$

Lymph node dissection 1-2 37

Lymphatic metastasis $\mathbf{1 - 2} 37$

Male breast cancer 5 266, 10542

Malignant glioma, overall survival 8-9 435

Malignant mesothelioma 10546

Mammotome 12653

Mastectomy 399

MDBF 399

Melanoma 7398

Melanoma metastases 5259

Melanoma, amelanotic 8-9 474

Melanoma, stage IV: surgical therapy, long-term survival 5259

Mesenteric alterations 10514

Mesothelioma 391

Metastases resection 5259

Metastasis 7 362, 7398

Metastatic breast cancer 8-9 447

Metastatic melanoma $\mathbf{7 3 8 0}$

Metronomic second-line chemotherapy 4185

Misty mesentery $\mathbf{1 0} 514$

Mitomycin C 5271

Molecular pathogenesis $\mathbf{1 0} 550$

Molecular studies 4179

Monoclonal antibodies $\mathbf{1 0} 550$
Morris' syndrome 1-2 53

Mouse model 391

Multicentre study $\mathbf{1 1} 605$

Multikinase inhibitors $\mathbf{7 3 9 8}$

Multiple myeloma 6335

Multivariate analysis $\mathbf{1 2} 679$

Mutation 4174, 11625

Myelodysplastic syndrome 8-9 477, 11629

Myelofibrosis 6325

Myeloma 1-2 45

Necrosis 12702

Neoadjuvant chemotherapy $\mathbf{7 3 6 6}$

Neoadjuvant treatment $\mathbf{1 2} 673$

Neoplasm metastasis, Liver $\mathbf{6} 328$

Neovagina formation 1-2 53

Neuroendocrine tumor $\mathbf{3} 119$

NK-cell lymphoma: Bone marrow involvement, CNS involvement 3115

Non-Hodgkin's lymphoma $\mathbf{1 0}$ 514, 11616

Non-small cell lung cancer $\mathbf{4} 185$

Non-small cell lung carcinoma $\mathbf{1 2} 665$

NSCLC 3123

NSCLC 4174

Olfactory 3119

Orbit $\mathbf{1 0} 529$

Orbital metastases 7387

Osteosarcoma $\mathbf{1 0} 535$

Ovarian carcinoma, metastasis: Breast, Rectum 4200

Ovarian yolk sac tumors $\mathbf{1 2} 679$

Oxaliplatin 5237

Paclitaxel 7 366, 8-9 435

Palliation $\mathbf{1 0} 529$

Pancreatic cancer 3 129, 5271

Papillary lesion, underestimation 12653

Penile neoplasms 1-2 37

Permanent brachytherapy 11599

Peutz-Jeghers syndrome 11625

Pilot study 4 185, 7362

Pinch off syndrome 7 404, 8-9 455

Pleural effusion 5262

Port catheter 8-9 455

Port device 7404

Port extravasation 7404

Port infection 7404

Port thrombosis 7 404, 8-9 455

Positron emission tomography (PET)

1-2 57, 3107
Preoperative radiotherapy $\mathbf{4} 166$

Primary adjuvant chemotherapy 6315

Primitive neuroectodermal tumor 4179

Prognosis 8-9 469, 10 529, 11 610, 12665

Prognostic factors $\mathbf{7 3 8 0 , 1 2} 679$

Programmed cell death $\mathbf{1 2} 702$

Prostate cancer 12697

Prostate 11599

Psychiatric morbidity 6343

Psycho-Oncology 11591

PTLD 12691

Quality of life $399,8-9$ 447, 8-9 477

Radiation 5266

Radiation therapy 3119

Radiochemotherapy: efficacy, prognostic factors, toxicity $\mathbf{5} 251$

Radiological findings 8-9 455

Radiotherapy 6 305, 6 309, 6 325, 6332 , 7 391, 10 529, 12657

Rectal GIST 12697

Radiotherapy planning 1-2 57

Rasburicase 4197

RECIST 3107

Rectal cancer 4166

Rectal carcinoma: Preoperative staging 5230

Rectal GIST 12697

Refractory disease $\mathbf{5} 237$

Regulatory T cells $\mathbf{1 - 2} 28,5243$

Renal failure, acute $\mathbf{4} 197$

Resource allocation $\mathbf{3} 85$

Response assessment $\mathbf{4} 166$

Retrobulbar metastases $\mathbf{1 0} 529$

Rhabdomyosarcoma 7391

S100B 7380

Score $\mathbf{1 0} 521$

Second Malignancy 5266

Serum CA-125 level 6315

SF-12 399

Sinonasal 3119

Small cell lung cancer 8-9 469

Soft tissue sarcoma 1-2 11

Soft tissue sarcoma, Response $\mathbf{3} 107$

Sorafenib 1-2 28, 7398

Stage IV 8-9 464

Staphylococcal scalded skin syndrome 11616

Statins $\mathbf{7 3 9 1}$

STK11 gene $\mathbf{1 1} 625$ 
Suicide gene therapy 391

Sunitinib 1-2 28

Surgery $\mathbf{6} 332, \mathbf{7} 391, \mathbf{1 2} 657$

Survival 12673

Survival rate $\mathbf{1 2} 679$

Survival: overall, progression-free $\mathbf{6} 309$

Symptoms 10529

Synchronous tumors $\mathbf{1 2} 697$

Tamoxifen 1-2 19

Target volume 1-2 57

Targeted therapy 4174, 10550

Telangiectasia, hereditary hemorrhagic 6328
Temozolomide 6309

Toxicity 8-9 435

Treatment modalities $\mathbf{1 0} 542$

Trisomy 22 8-9 440

Tumor lysis syndrome, acute $\mathbf{4} 197$

Tumor markers $\mathbf{7 3 8 0}$

Tumor osteolysis $\mathbf{1 0} 521$

Tumors 7362

Tumor-treating fields (TTFields) 7362

Tyrosin kinase inhibitors $\mathbf{1 0} 550$

Tyrosine kinase 4174

Univariate analysis $\mathbf{1 2} 679$

Urinary bladder neovagina $\mathbf{1 - 2} 53$
Vacuum-assisted breast biopsy $\mathbf{1 2} 653$

Vaginal melanoma 8-9 474

Valproic acid $\mathbf{1 1} 629$

VEGF 10535

Venous thromboembolism 3129

Vessel rupture 5262

Vinorelbine 8-9 447

Vulvar cancer 6332

Years of life gained $\mathbf{3} 85$

Yttrium-90 1-2 49 